\title{
Environmental TEM Study of Electron Beam Induced Sintering of Ag Nanoparticles
}

\author{
Yuzi Liu ${ }^{1}$, Yugang Sun ${ }^{1}$ \\ ${ }^{1}$ Center for Nanoscale Materials, Argonne National Laboratory, 9700 S. Cass Ave. Argonne, IL, United \\ States.
}

Sintering process of nanoparticles represents an interesting topic in maintaining the stability of colloidal nanoparticles under applications as catalyst and synthesizing hybrid multiple functional nanomaterials. In this presentation, we will report the sintering of $\mathrm{Ag}$ nanoparticles studied by in-situ transmission electron microscopy (TEM) under different environments as vacuum, air and $\mathrm{N}_{2}$ in a JEOL $2100 \mathrm{~F}$ working at $200 \mathrm{kV}$. An air cell made from two $\mathrm{Si}_{3} \mathrm{~N}_{4}$ chips was applied to seal the $\mathrm{Ag}$ nanoparticles with $\mathrm{N}_{2}$ and air. In order to eliminate the sample vibration from stiff metal gas tubing, the gas tubing was removed once the sample was flushed by air or $\mathrm{N}_{2}$. So, there is no gas flowing during electron beam irradiation on the Ag nanoparticles. The electron beam conditions was remain the same as possible as we can.

Figure 1 shows the image serials of Ag nanoparticles sintering process in vacuum. Obviously, that the smaller particle is more active than the bigger ones since the diffusion evolve faster on the smaller particles (from 10mins to $25 \mathrm{mins}$ in Fig. 1). With the time going, the small particle diffused to the bigger ones eventually as shown as 30mins in Fig1. The size of the merged particle keep decreasing under the electron beam irradiation from $30 \mathrm{mins}$ to $60 \mathrm{mins}$. This means the sublimation process under electron beam irradiation.

Figure 2 and 3 show the Ag nanoparticle sintering process by electron beam irradiation in 1 atmosphere of air and $\mathrm{N}_{2}$ respectively. We can find the sintering process is almost same as which in vacuum. We found the sintering evolving is faster in $\mathrm{N}_{2}$ than in air since we found there is more perfect triangle shape at 25 mins in $\mathrm{N}_{2}$. The surface of the nanoparticles maybe oxidized in air under electron beam irradiation. It will block the Ag interdiffusion between the nanoparticles and result in the slower sintering. We can't compare the sintering speed with the case in vacuum since the Ag nanoparticles were deposited on ultrathin carbon grid. The further experiments will use $\mathrm{Si}_{3} \mathrm{~N}_{4}$ grid in vacuum and map the oxygen distribution on the sintered nanoparticles.

[1] This work was performed at the Center for Nanoscale Materials, a U.S. Department of Energy, Office of Science, Office of Basic Energy Sciences User Facility under Contract No. DE-AC02$06 \mathrm{CH} 11357$. 

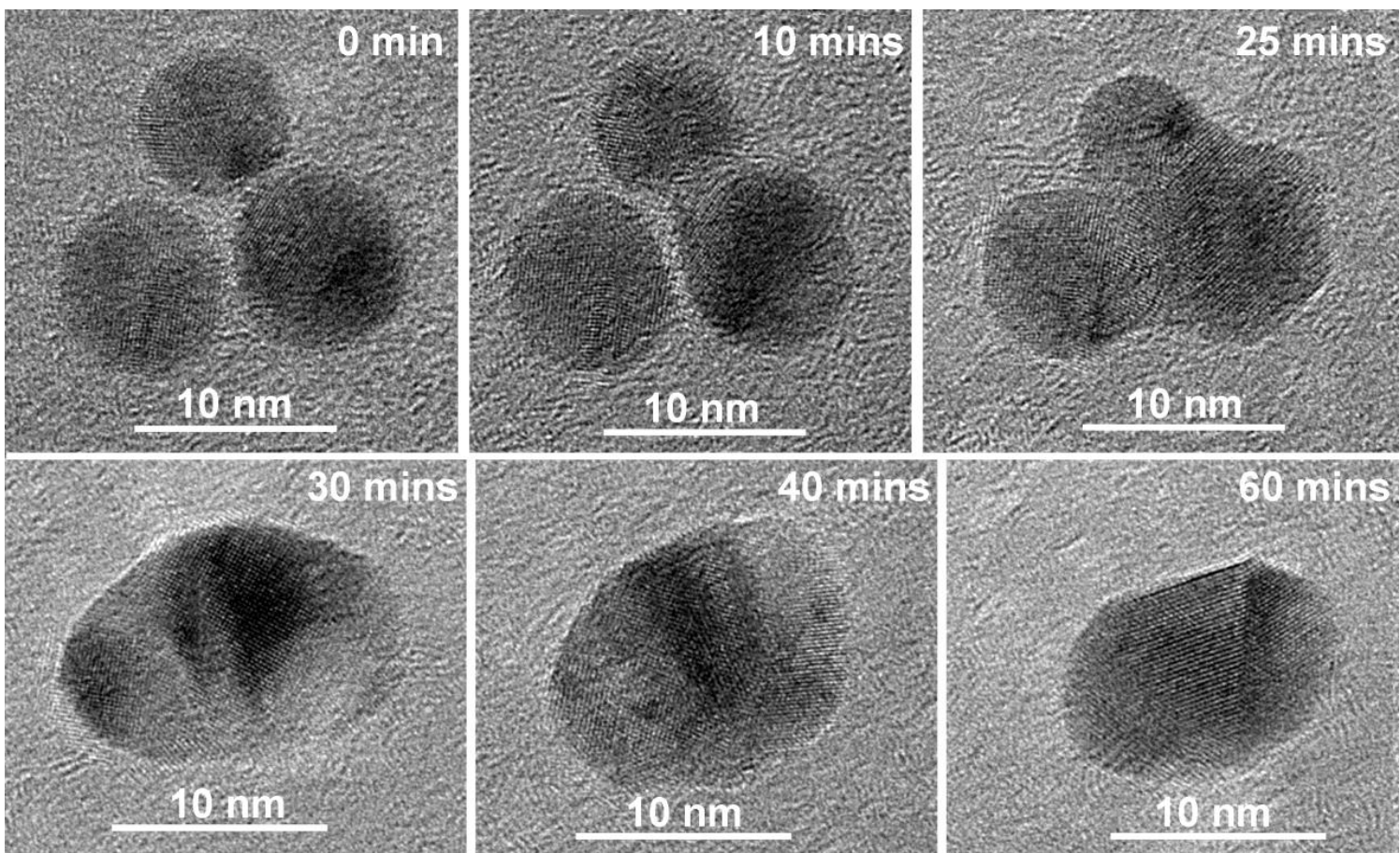

Figure 1. Ag nanoparticles sintering progress along with time.
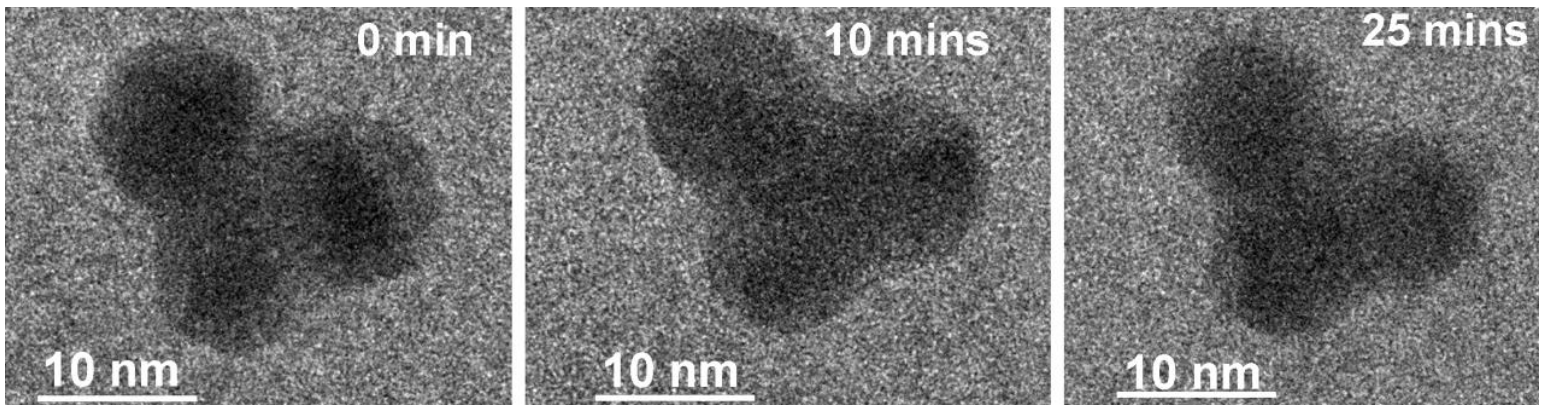

Figure 2. Ag nanoparticles sintering progress along with time in air at 1 atmosphere.
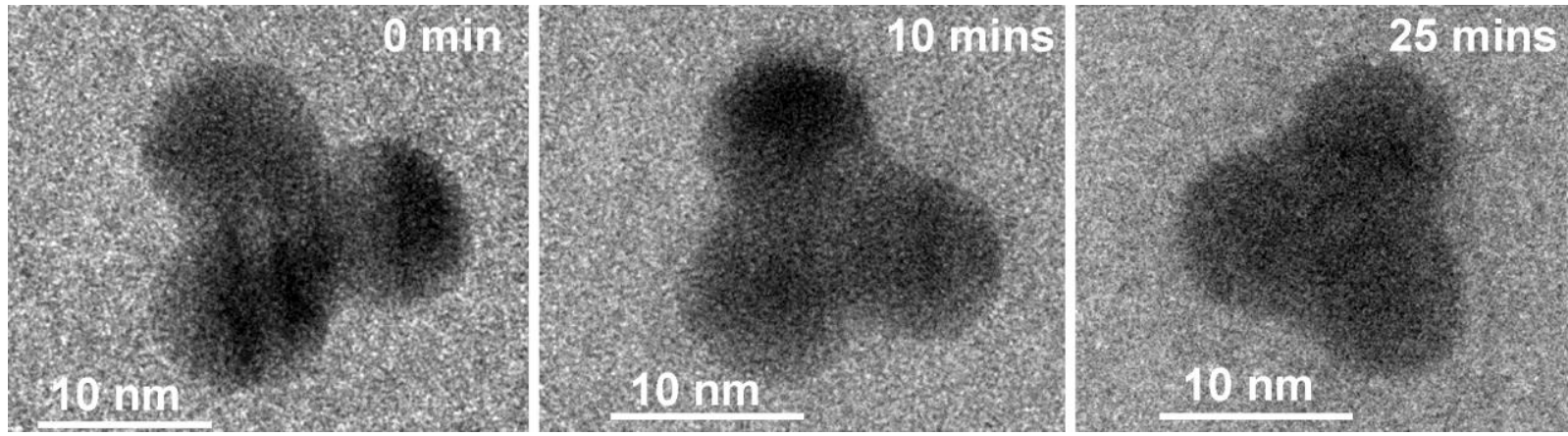

Figure 3. Ag nanoparticles sintering progress along with time in $\mathrm{N}_{2}$ at 1 atmosphere. 